\title{
L'acier en France en 2010 Rapport annuel de la Fédération Française de l'Acier (FFA)
}

Les prévisions d'un optimisme réservé, formulées dans notre rapport d'activité 2009, se sont vues confirmées par les faits. Nous pouvons considérer que l'année 2010 aura constitué, notamment pour l'industrie française de l'acier, une année de convalescence, sur le chemin d'un retour possible au niveau des années fastes d'avant la crise économique. Presque la moitié de ce parcours qui sera probablement plus long que prévu, a en effet été accompli en 2010, avec une progression de $20 \%$ de la production française d'acier brut (à comparer à $+23,9 \%$ au niveau européen), production qui a atteint un niveau de 15,4 millions de tonnes soit encore 22,3 \% en deçà de la production annuelle moyenne sur la période 2003-2007.

La consommation réelle d'acier a également progressé en 2010 de $7 \%$ en France à comparer aux $6 \%$ pour l'Union européenne mais avec de grandes disparités de performances entre pays du nord et du sud.

Il convient de noter que ces progressions significatives s'inscrivent dans un paysage de croissance économique européenne relativement limitée à $1,8 \%$, la croissance économique française $(1,5 \%)$ se situant légèrement en dessous de la moyenne européenne.

Si l'on étend son champ de vision à l'ensemble de la sidérurgie mondiale, l'année 2010 aura été marquée par des faits bien plus majeurs avec:

- un record de production d'acier brut avec 1412 millions de tonnes en progression de près de $15 \%$ par rapport à 2009 ;

- des records de production nationale d'acier brut pour la Chine avec 627 millions de tonnes, soit 44,4 \% de la production mondiale, et pour l'Inde à 66,8 millions de tonnes, soit 4,7\% de la production mondiale;

- l'arrivée de six producteurs chinois dans le «top 10 » des producteurs mondiaux d'acier brut, le rang $n^{\circ} 1$ étant toujours détenu par ArcelorMittal dont la production annuelle (90,5 millions de tonnes) aura représenté 6,4\% de la production mondiale, les deux japonais Nippon Steel Corporation et JFE et le coréen Posco complétant ce « top $10 »$;

- La flambée du prix des matières premières avec des augmentations d'environ $90 \%, 75 \%, 50 \%$ et $30 \%$ sur l'année, respectivement pour le minerai de fer, le coke métallurgique, les ferrailles ou le nickel.

Que conviendra-t-il de retenir pour notre industrie en 2010 ?

En tout premier lieu, il faut souligner la flexibilité de notre industrie, démontrée par sa capacité à s'adapter à la demande réelle du marché et à redémarrer ou à ralentir, dans un délai relativement court, ses outils de production, notamment les hauts-fourneaux. Il convient de souligner également sa capacité à faire face aux variations très fortes des prix des matières premières, éléments essentiels du coût d'élaboration de l'acier. Enfin, il conviendra de noter la stabilisation du niveau d'emploi dans notre secteur après une année très difficile en la matière, en 2009.

L'année 2011 devrait voir l'industrie de l'acier se maintenir sur une pente positive avec de nouvelles progressions des consommations réelle et apparente d'acier, tant au niveau européen, où les prévisions sont respectivement de $+5 \%$ et $+6 \%$, qu'au niveau français, avec des prévisions un peu en dessous, à un niveau de l'ordre de $+3 \%$. Ces prévisions sont confortées par celles des principaux secteurs utilisateurs d'acier : l'automobile, les industries mécaniques et le secteur de la construction (bâtiment, travaux publics). Il faut espérer que les catastrophes naturelles et les évènements géopolitiques survenus en début d'année ne viendront pas entamer l'optimisme affiché en début d'année 2011, confirmé par les résultats du premier trimestre. 


\section{Le marché}

L'année 2010 aura été celle d'une consolidation de la reprise économique mondiale amorcée au cours de la seconde partie de l'année 2009. Cependant, cette année aura également été caractérisée par de fortes turbulences en Europe avec la crise de la dette publique qui a touché plusieurs pays et a été source d'inquiétudes dans la zone européenne. La croissance mondiale a dépassé sa moyenne de longue période, atteignant un rythme de 4,7\% mais avec de grandes disparités selon les régions du monde. Cependant, si l'économie mondiale a renoué avec l'expansion, l'intensité de la reprise a divergé.

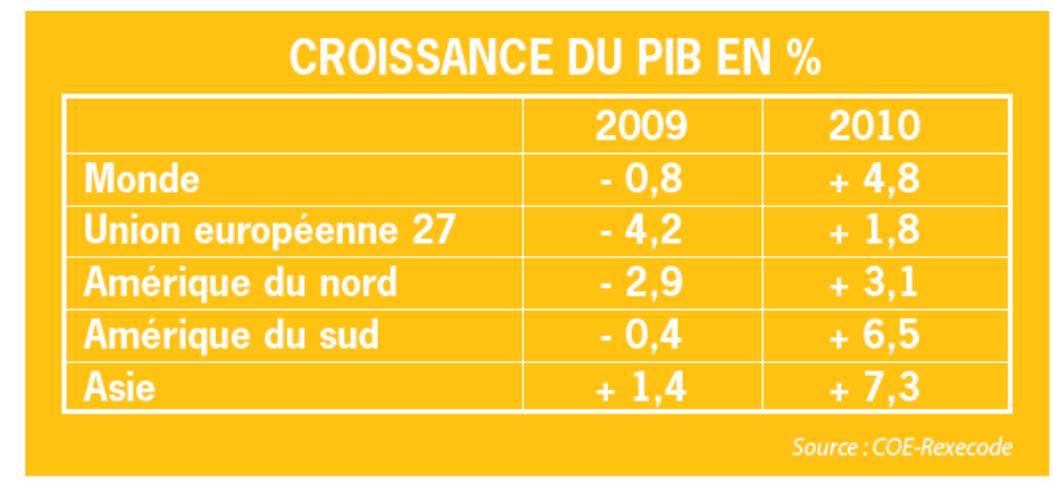

Dans un premier groupe, on retrouve les pays émergents de la zone asiatique mais également le Brésil qui ont rapidement dépassé leur niveau d'avant la crise contrairement aux États-Unis et à l'Europe qui ont enregistré des croissances moindres et n'ont pas retrouvé les niveaux de production d'avant la crise.

En Europe, la croissance a été modérée avec de fortes distorsions de performance entre les pays membres alimentées par la situation des finances publiques dégradée dans plusieurs pays. Parmi les grands pays, l'Espagne a été très affectée par les tensions sur le financement de la dette publique et sa croissance a été sensiblement négative, conséquence également de la crise de son secteur immobilier. À l'opposé, l'Allemagne s'est distinguée par sa très bonne performance avec une croissance de 3,5\% qui compense cependant sa récession marquée en 2009, conséquence de sa forte exposition à la demande internationale. La croissance économique de la France a été proche de son évolution tendancielle et de la moyenne de la zone euro, enregistrant un rythme de 1,5\% en 2010. La bonne tenue de la consommation des ménages a été un des principaux facteurs de soutien tandis que les performances à l'exportation ont été modestes.

L'activité des secteurs utilisateurs d'acier dans l'UE a progressé de près de $6 \%$ après un effondrement de près de $20 \%$ en 2009, la France se situant sensiblement en dessous de la moyenne européenne.

Les immatriculations de voitures particulières et de véhicules commerciaux légers en UE ont baissé de $4 \%$, bien qu'elles aient été soutenues par les primes à la casse encore en vigueur une partie de l'année dans plusieurs pays. En France, elles ont été stables soutenues par une prime maintenue tout au long de l'année. La production européenne de l'ensemble du secteur automobile a fortement rebondi, atteignant un rythme de $20 \%$ après un effondrement l'année précédente. Cette reprise a été accentuée par l'ajustement des stocks de véhicules dans l'ensemble de la filière.

La reprise du secteur de la construction mécanique et du travail des métaux a été plus modeste et a atteint près de $10 \%$, grâce à de bonnes performances à l'exportation en particulier de l'Allemagne, mais également une reprise des investissements en Europe dans la seconde partie de l'année. L'activité reste cependant en retrait de près de $20 \%$ par rapport à son niveau atteint en 2007.

Autre important secteur consommateur d'acier, celui de la construction en Europe a enregistré une évolution très différenciée de celle des secteurs précédents et a baissé de $2 \%$, la France enregistrant une évolution voisine de cette moyenne. Cependant la situation a été contrastée entre les pays de la zone européenne, l'Espagne a encore enregistré un effondrement dépassant $12 \%$, l'activité de la construction espagnole affichait un retrait de près de $40 \%$ par rapport à son niveau de 2007 tandis que le secteur ne restait en recul que de $10 \%$ en Union européenne.

La consommation réelle d'acier a par conséquent enregistré un rebond voisin de $7 \%$ en Europe et en France. La consommation apparente a enregistré une hausse de $23 \%$ en Europe et de $18 \%$ en France, hausse qui n'a pas généré 
une montée des stocks d'acier qui sont restés à des niveaux bas au regard du passé dans l'ensemble de la chaîne de consommation en fin d'année.

La reprise de la consommation apparente d'acier a, d'une façon générale, été plus intensive dans les aciers plats qui ont progressé de $23 \%$ tandis que celle des produits longs n'a été que de $11 \%$ en France.

MARCHÉ INTÉRIEUR DES PRODUITS FINIS SIDÉRURGIQUES
(en milliers de tonnes)
\begin{tabular}{|l|c|c|}
\hline Approvisionnement & 2010 & Variation 2010/2009 en \% \\
\hline Livraisons des usines françaises & 5682 & 13,5 \\
\hline Importations & 8597 & 24,7 \\
\hline TOTAL & 14279 & 20,0 \\
\hline
\end{tabular}

\section{Le commerce extérieur de l'Union européenne}

Les importations de produits sidérurgiques de l'UE en provenance des pays tiers ont accéléré de $30 \%$, notamment les importations de produits plats en liaison avec le rebond de la consommation apparente d'acier, ceci après un effondrement voisin de $50 \%$ l'année précédente. Les importations en provenance de Chine ont rebondi sans rejoindre cependant leur niveau d'avant la crise. La Russie et l'Ukraine conservent une part importante des flux d'importations, voisine de $50 \%$.

Les exportations de produits sidérurgiques vers les pays tiers ont enregistré une croissance qui a effacé la baisse de 2009.

Au total, la balance commerciale est restée légèrement positive.

\section{Le commerce extérieur français}

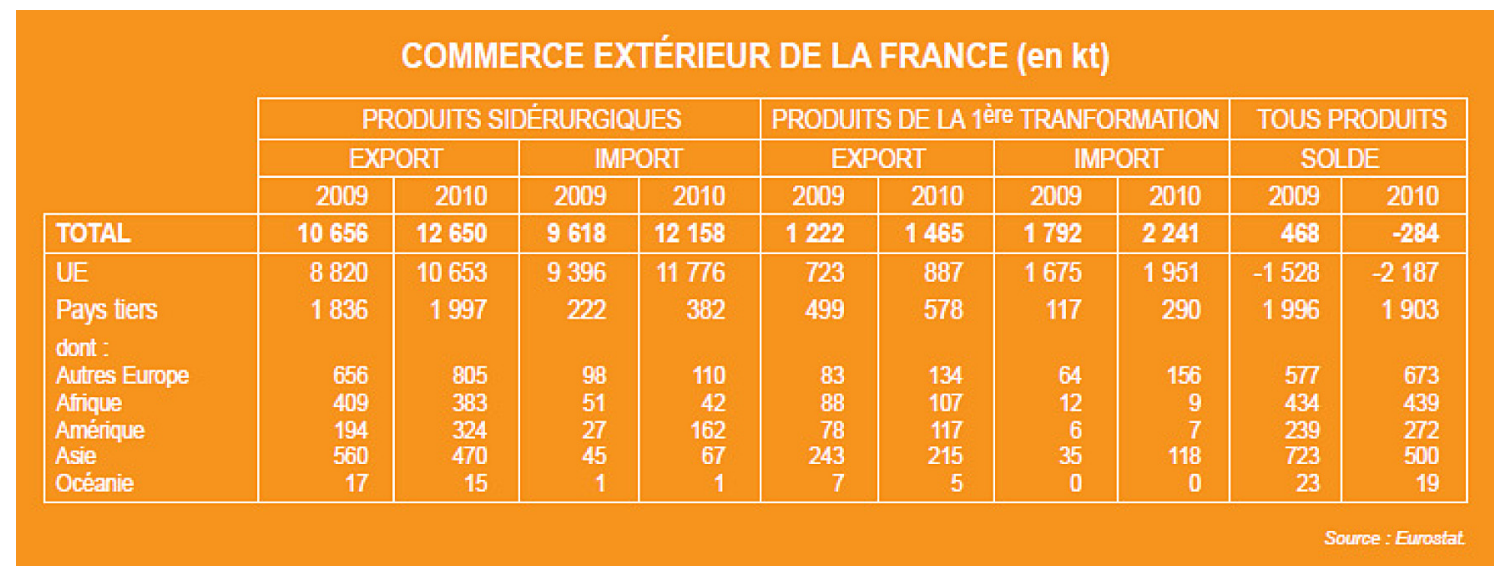

Les importations françaises de produits sidérurgiques et de produits de la première transformation ont progressé de $25 \%$, en corrélation avec le rebond de la consommation d'acier.

Les exportations françaises de produits sidérurgiques et de produits de la première transformation ont également augmenté de $19 \%$ en corrélation avec le rebond de la consommation dans les pays européens partenaires.

Le solde des échanges extérieurs de produits sidérurgiques et de produits de la première transformation s'est dégradé et a été négatif pour les produits issus de la première transformation. 


\section{Production mondiale d'acier}

La production mondiale d'acier a poursuivi son rebond amorcé à fin du premier semestre 2009, et a progressé de $15 \%$ l'année dernière.

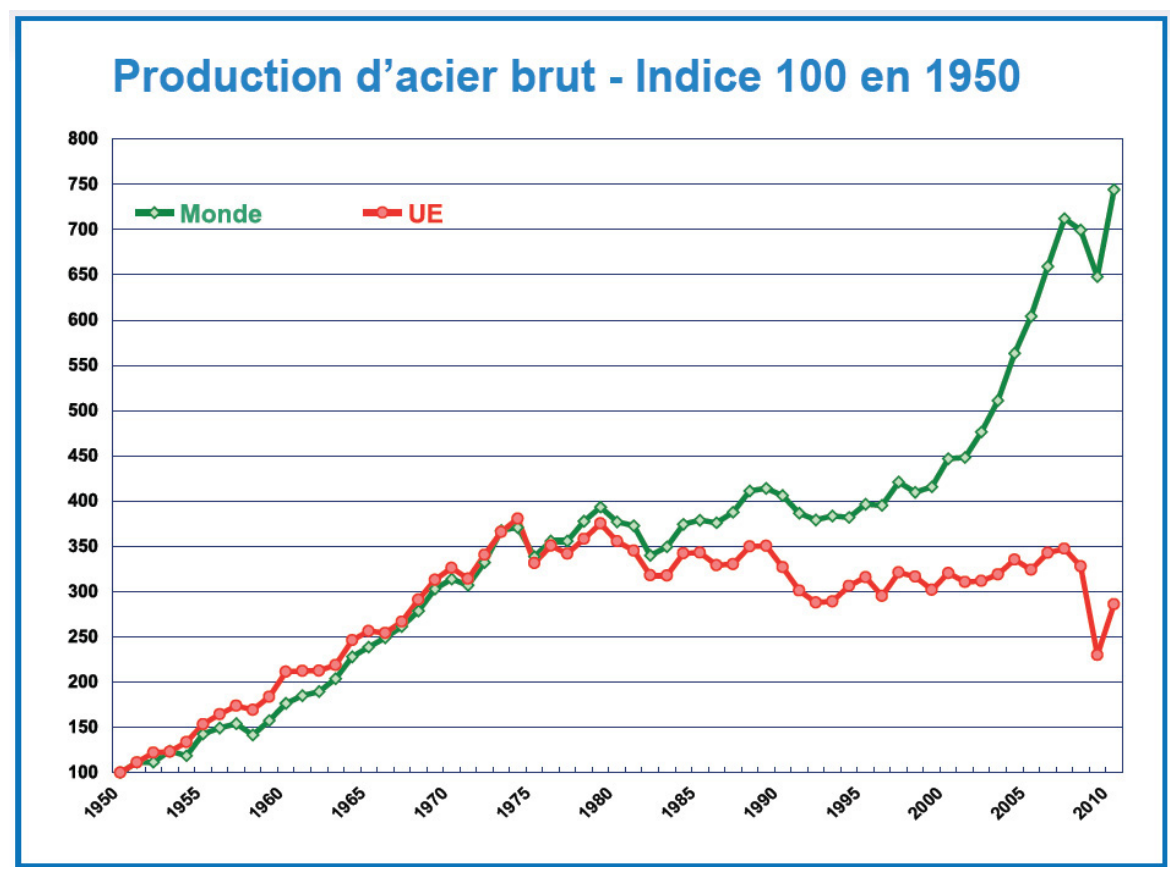

Dans l'Union européenne, la progression a atteint un rythme de $24 \%$ et de $20 \%$ en France. La production d'acier reste cependant en retrait de $20 \%$ en France par rapport à son niveau atteint en 2007 et de $18 \%$ pour l'Union européenne. La part de l'Union européenne dans la production mondiale d'acier poursuit sa baisse et représentait $12 \%$ en 2010.

En Amérique du Nord, la reprise a été forte avec un taux de progression de $35 \%$, le niveau reste en recul de $18 \%$ par rapport à 2007. La croissance de la production en Amérique du sud a atteint 16\% et celle du Brésil $24 \%$.

L'Asie, dont la part dans la production d'acier dans le monde a atteint $64 \%$ en 2010, et celle de la Chine $44 \%$, a continué sa progression sur un rythme de $12 \%$.

\section{Évolution de la production mensuelle d'acier brut (en kt CVS)}

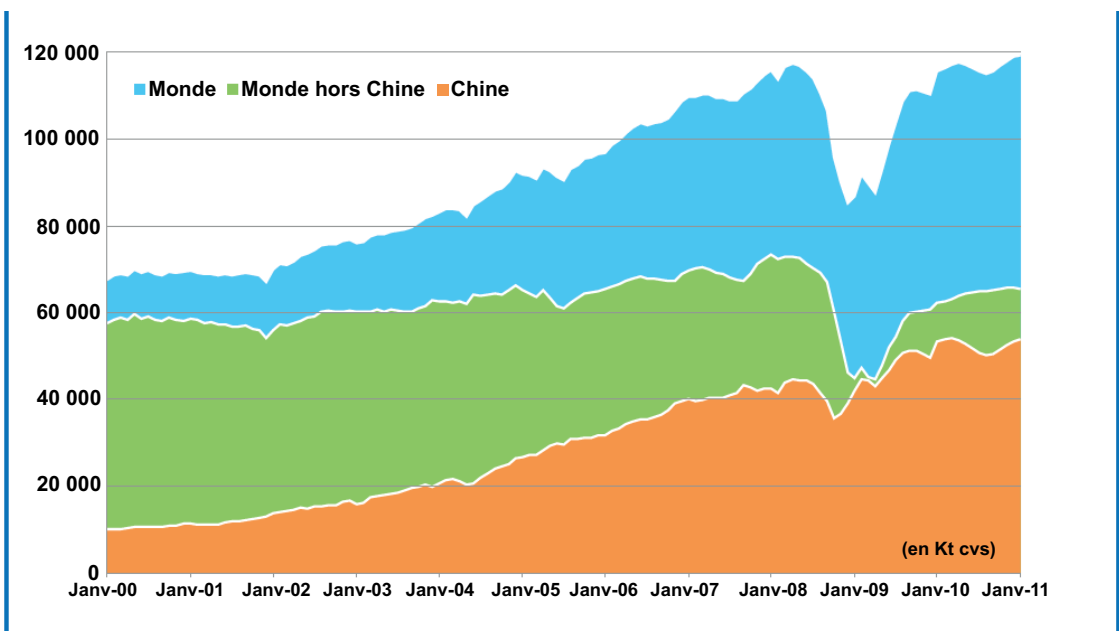




\section{LA PRODUCTION MONDIALE D'ACIER}

\begin{tabular}{|c|c|c|c|c|c|c|}
\hline & \multicolumn{3}{|c|}{ PRODUCTION (kt) } & \multicolumn{2}{|c|}{ VARIATIONS } \\
\hline & & 2008 & 2009 & 2010 & $2009 / 2008$ & $2010 / 2009$ \\
\hline \multicolumn{2}{|c|}{ Union européenne } & 198195 & 139366 & 172617 & $-29,7 \%$ & $23,9 \%$ \\
\hline dont : & $\begin{array}{l}\text { Allemagne } \\
\text { Espagne } \\
\text { France } \\
\text { Italie } \\
\text { Royaume-Uni } \\
\text { Pologne }\end{array}$ & \begin{tabular}{r|r|}
45833 \\
18640 \\
17878 \\
30590 \\
13521 \\
9728
\end{tabular} & $\begin{aligned} 32670 \\
14358 \\
12840 \\
19848 \\
10079 \\
7128\end{aligned}$ & $\begin{array}{r}43830 \\
16343 \\
15414 \\
25744 \\
9709 \\
7993\end{array}$ & $\begin{array}{l}-28,7 \% \\
-23,0 \% \\
-28,2 \% \\
-35,1 \% \\
-25,5 \% \\
-26,7 \%\end{array}$ & $\begin{array}{l}+34,2 \% \\
+13,8 \% \\
+20,0 \% \\
+29,7 \% \\
-3,7 \% \\
+12,1 \%\end{array}$ \\
\hline \multicolumn{2}{|c|}{$\begin{array}{l}\text { Autres pays } \\
\text { d'Europe de l'ouest }\end{array}$} & 29928 & 29106 & 33605 & $-2,7 \%$ & $+15,5 \%$ \\
\hline dont : & Turquie & 26806 & 25304 & 29143 & $-5,6 \%$ & $+15,2 \%$ \\
\hline \multicolumn{2}{|c|}{$\begin{array}{l}\text { Autres pays } \\
\text { d'Europe de l'est }\end{array}$} & 114345 & 97645 & 108228 & $-14,6 \%$ & $+10,8 \%$ \\
\hline dont: & Russie & 68510 & 60011 & 66942 & $-12,4 \%$ & $+11,5 \%$ \\
\hline \multicolumn{2}{|c|}{ Amérique du nord } & 124494 & 82578 & 111406 & $-33,7 \%$ & $+34,9 \%$ \\
\hline dont & États-Unis & 91350 & 58196 & 80495 & $-36,3 \%$ & $+38,3 \%$ \\
\hline \multicolumn{2}{|c|}{ Amérique du sud } & 47354 & 37775 & 43765 & $-20,2 \%$ & $+15,9 \%$ \\
\hline dont: & Brésil & 33716 & 26506 & 32820 & $-21,4 \%$ & $+23,8 \%$ \\
\hline \multicolumn{2}{|l|}{ Asie } & 771013 & 804862 & 897913 & $+4,4 \%$ & $+11,6 \%$ \\
\hline \multicolumn{2}{|r|}{$\begin{array}{l}\text { Corée } \\
\text { Inde } \\
\text { Japon }\end{array}$} & $\begin{array}{r}500312 \\
53625 \\
57791 \\
118739\end{array}$ & \begin{tabular}{r|}
573567 \\
48572 \\
62838 \\
87534
\end{tabular} & $\begin{array}{r}626654 \\
58363 \\
66848 \\
109599\end{array}$ & $\begin{array}{r}+14,6 \% \\
-9,4 \% \\
+8,7 \% \\
-26,3 \%\end{array}$ & $\begin{array}{r}+9,3 \% \\
+20,2 \% \\
+6,4 \% \\
+25,2 \%\end{array}$ \\
\hline \multicolumn{2}{|c|}{ Moyen Orient } & 16646 & 17656 & 19590 & $+6,1 \%$ & $+11,0 \%$ \\
\hline \multicolumn{2}{|c|}{ Afrique } & 16997 & 15170 & 16621 & $-10,7 \%$ & $+9,6 \%$ \\
\hline \multicolumn{2}{|c|}{ Australie - Nouvelle Zélande } & 8424 & 6014 & 8149 & $-28,6 \%$ & $+35,5 \%$ \\
\hline \multicolumn{2}{|c|}{ Monde } & 1327396 & 1230172 & 1411894 & $-7,3 \%$ & $+14,8 \%$ \\
\hline
\end{tabular}




\section{Production mondiale d'acier brut - Année 2010}

$12,2 \%$ Union européenne
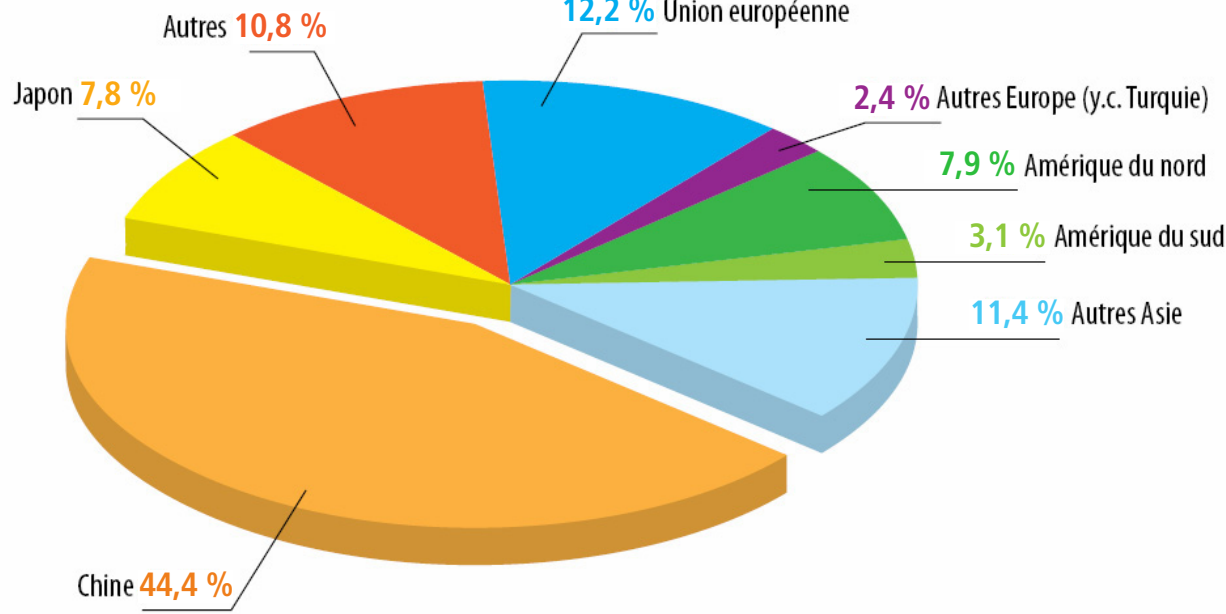

\section{Production d'acier brut}

\section{Union européenne à 27 - Année 2010}

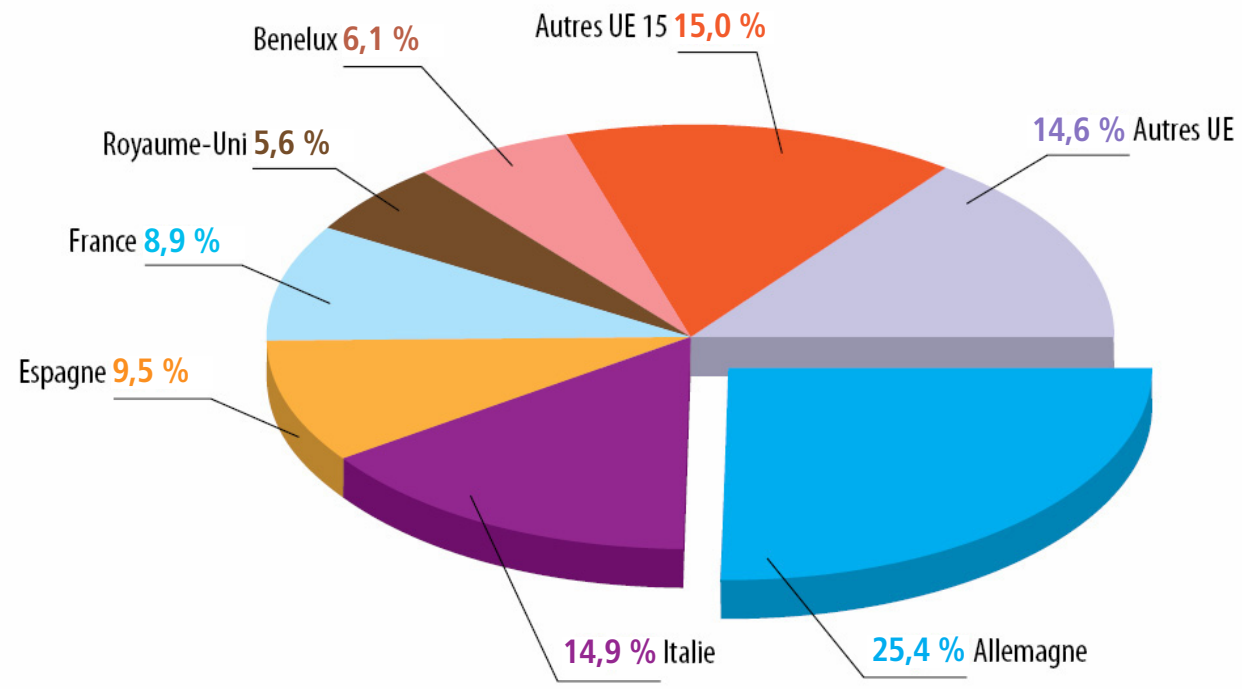




\section{L'acier en France en 2010}

\section{Approvisionnements}

\section{Minerai de fer}

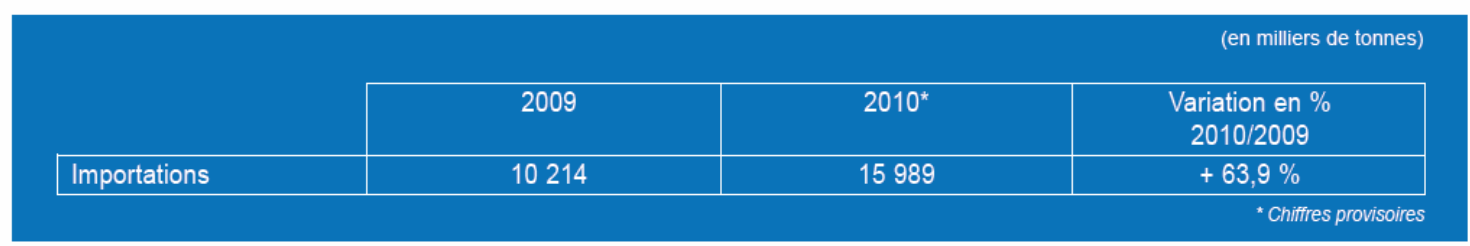

\section{Principales sources d'approvisionnement à l'importation}

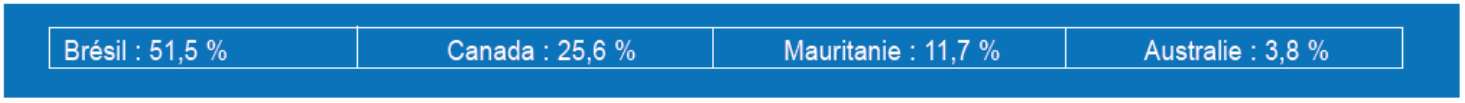

\section{Ferrailles en sidérurgie}

\begin{tabular}{|l|c|c|c|}
\cline { 2 - 4 } \multicolumn{1}{c|}{} & 2009 & $2010^{*}$ & $\begin{array}{c}\text { Variation en \% } \\
2010 / 2009\end{array}$ \\
\hline Collecte nationale & 6221 & 7190 & $15,6 \%$ \\
\hline Importations & 1505 & 1404 & $-6,7 \%$ \\
\hline Approvisionnement total & 7726 & 8594 & $11,2 \%$ \\
\hline Consommation & 7294 & 8103 & $11,1 \%$ \\
\hline
\end{tabular}

\section{Énergie}

\begin{tabular}{|l|c|}
\hline \multicolumn{2}{|c|}{$\begin{array}{c}\text { Consommation d'énergie 2010 } \\
\text { (hors gaz sidérurgiques) } \\
\text { (Variation par rapport à l'année 2009) }\end{array}$} \\
\hline Coke et houille & $+25,6 \%$ \\
\hline Fuels & $-11,3 \%$ \\
\hline Gaz naturel & $+22,8 \%$ \\
\hline Electricité & $+17,6 \%$ \\
\hline
\end{tabular}

Les évolutions constatées dans la consommation d'énergie s'expliquent essentiellement par deux facteurs :

- l'augmentation de 20,1\% de la production d'acier par rapport à 2009, au creux de la crise ;

- l'augmentation de la part des aciéries de conversion qui ont produit 63,7 \% de l'acier en 2010 contre 59,8 \% en 2009, cette évolution étant liée principalement à l'arrêt de l'aciérie électrique de Gandrange et à une marche très ralentie des hauts-fourneaux lorrains d'ArcelorMittal en 2009. 


\section{Répartition des consommations d'énergie en TEP}

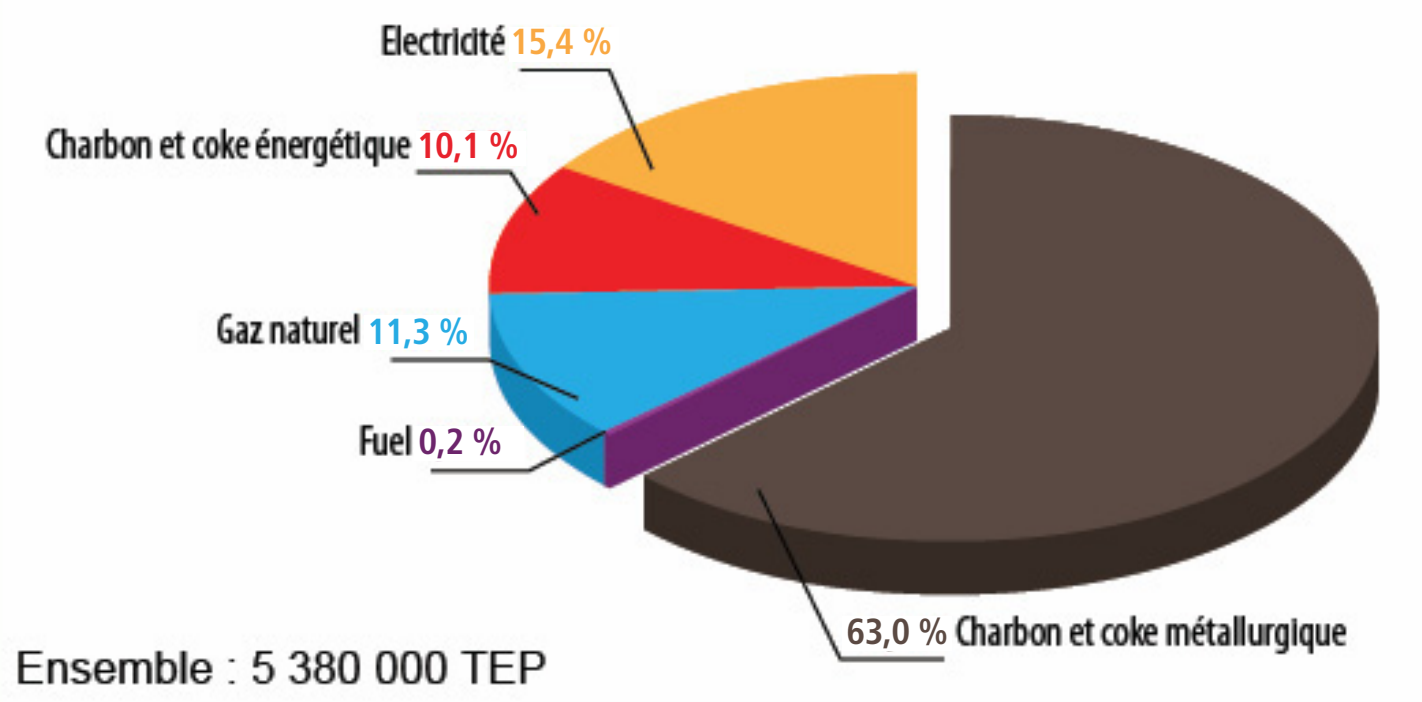

\begin{tabular}{|c|c|}
\hline Production d'acier brut & 15,4 millions de tonnes \\
\hline Part de la production issue de la filière électrique & $36,3 \%$ \\
\hline Part des produits issus de la coulée continue & $95,9 \%$ \\
\hline $\begin{array}{l}\text { Livraison de produits sidérurgiques } \\
\text { (hors relaminage en France et travail à façon) }\end{array}$ & 16,9 millions de tonnes \\
\hline Activité sidérurgique & 11,5 milliards d'euros \\
\hline $\begin{array}{r}\text { Commerce extérieur : exportations } \\
\text { importations }\end{array}$ & $\begin{array}{l}9,2 \text { milliards d'euros } \\
8,8 \text { milliards d'euros }\end{array}$ \\
\hline $\begin{array}{l}\text { Consommation française de produits sidérurgiques } \\
\text { (consommation apparente) }\end{array}$ & 12,9 millions de tonnes \\
\hline $\begin{array}{l}\text { Livraisons totales de produits } \\
\text { de la première transformation de l'acier }\end{array}$ & 2,5 millions de tonnes \\
\hline
\end{tabular}

\section{Les chiffres-clés}

NB. Le rapport 2010 complet, ainsi que les rapports des années précédentes, sont disponibles sur le site www.acier.org Une version en couleurs est disponible à l'adresse suivante : www.revue-metallurgie.org 